\title{
On the Matrix Versions of Incomplete Extended Gamma and Beta Functions and Their Applications for the Incomplete Bessel Matrix Functions
}

\author{
Chaojun Zou, ${ }^{1,2}$ Mimi Yu, ${ }^{3}$ Ahmed Bakhet $\mathbb{D}^{4,5}$ and Fuli $\mathrm{He}^{5}$ \\ ${ }^{1}$ College of Economics and Management, Changsha Normal University, Changsha 410148, China \\ ${ }^{2}$ College of Mechanical and Electrical Engineering, Hunan Agricultural University, Changsha 410128, China \\ ${ }^{3}$ School of Mathematics and Statistics, Ningxia University, Yinchuan 750021, China \\ ${ }^{4}$ Department of Mathematics, Faculty of Science, Al-Azhar University, Assiut 71524, Egypt \\ ${ }^{5}$ School of Mathematics and Statistics, Central South University, Changsha 410083, China \\ Correspondence should be addressed to Ahmed Bakhet; kauad_2006@csu.edu.cn
}

Received 15 January 2021; Accepted 18 February 2021; Published 6 December 2021

Academic Editor: Eric Campos-Canton

Copyright (C) 2021 Chaojun Zou et al. This is an open access article distributed under the Creative Commons Attribution License, which permits unrestricted use, distribution, and reproduction in any medium, provided the original work is properly cited.

\begin{abstract}
In this paper, we first introduce the incomplete extended Gamma and Beta functions with matrix parameters; then, we establish some different properties for these new extensions. Furthermore, we give a specific application for the incomplete Bessel matrix function by using incomplete extended Gamma and Beta functions; at last, we construct the relation between the incomplete confluent hypergeometric matrix functions and incomplete Bessel matrix function.
\end{abstract}

\section{Introduction}

In many areas of applied mathematics, various types of special functions have become essential tools for scientists and engineers. The continuous development of mathematical physics, probability theory, and other areas has led to new classes of special functions and their extensions and generalizations (see [1-7]). Generalizations of the classical special functions to matrix setting have become important during last years. Special matrix functions appear in solutions for some physical problems. Applications of special matrix functions also grow and have become active areas in recent literature including statistics, Lie groups theory, and differential equations (see, e.g., [8-11] and elsewhere). New extensions of some of the well-known special matrix functions such as gamma matrix function, beta matrix function, and Gauss hypergeometric matrix function have been extensively studied in recent papers [12-19]. Our main purpose in this paper is to obtain an extension of the incomplete Gamma and Beta matrix functions and will be introduced as application to incomplete Bessel functions with matrix coefficients.
The structure of this paper is as follows. In Section 2, we give basic definitions and preliminaries that are needed in the subsequent sections. In Section 3, we define the generalized incomplete Gamma function with matrix coefficients and study to some properties of the generalized incomplete Gamma matrix function. In Section 4, we present the Beta matrix functions and consider some properties of the incomplete Beta matrix function. Finally, in Section 5, we consider application of the incomplete Bessel matrix function by using incomplete Beta and incomplete confluent hypergeometric matrix function.

\section{Preliminaries and Basic Definitions}

Throughout this paper, $I$ and $\mathbf{0}$ will denote the identity matrix and null matrix in $\mathbb{C}^{r \times r}$, respectively. For a matrix $A \in \mathbb{C}^{r \times r}$, its spectrum is denoted by $\sigma(A)$. We say that if $\operatorname{Re}(\xi)>0$, for all $\xi \in \sigma(A)$, a matrix $A$ in $\mathbb{C}^{r \times r}$ is a positive stable matrix, where $\sigma(A)$ is the set of all eigenvalues of $A$. In $[16,20]$, if $f(z)$ and $g(z)$ are holomorphic functions in an open set $\Lambda$ of the complex plane and if $A$ is a matrix in $\mathbb{C}^{r \times r}$ 
for which $\sigma(A) \subset \Lambda$, then $f(A) g(A)=g(A) f(A)$. The logarithmic norm of a matrix $A$ in $\mathbb{C}^{r \times r}$ is defined as (see $[17,21])$

$$
\mu(A)=\lim _{h \longrightarrow 0} \frac{\|I+h A\|-1}{h}=\max \left\{z: z \in \sigma\left(\frac{A+A^{*}}{2}\right)\right\} .
$$

Suppose the number $\widetilde{\mu}(A)$ is such that

$$
\widetilde{\mu}(A)=-\mu(-A)=\min \left\{z: z \in \sigma\left(\frac{A+A^{*}}{2}\right)\right\} .
$$

For all $A$ in $\mathbb{C}^{r \times r}$ and

$$
A+n I \text { is invertible for all integers } n \text {, }
$$

then the Pochhammer symbol is defined by see, e.g., $[8,22]$ :

$$
\begin{aligned}
(A)_{n} & =A(A+I), \ldots,(A+(n-1) I) \\
& =\Gamma(A+n I) \Gamma^{-1}(A) ; \quad(A)_{0} \equiv I .
\end{aligned}
$$

Definition 1 (see [21]). Let $A$ be a positive stable matrix in $\mathbb{C}^{r \times r}$ and $x$ be a positive real number. Then, the incomplete Gamma matrix function $\gamma(A, x)$ and its complement $\Gamma(A, x)$ are defined by

$$
\begin{aligned}
& \gamma(A, x)=\int_{0}^{x} e^{-t} t^{A-I} \mathrm{~d} t, \quad t^{A-I}=\exp ((A-I) \ln t), \\
& \Gamma(A, x)=\int_{x}^{\infty} e^{-t} t^{A-I} \mathrm{~d} t, \quad t^{A-I}=\exp ((A-I) \ln t),
\end{aligned}
$$

respectively, which satisfy the following decomposition formula (see [21]):

$$
\gamma(A, x)+\Gamma[A, x]=\Gamma(A) .
$$

By inserting a regularization matrix factor $e^{-(B / t)}, B \in \mathbb{C}^{r \times r}$, Abul-Dahab and Bakhet [13] have introduced the following generalization of the gamma matrix function.

Definition 2. Let $A$ and $B$ be positive stable matrices in $C^{r \times r}$; then, the generalized Gamma matrix function $\Gamma(A, B)$ is defined by

$$
\Gamma(A, B)=\int_{0}^{\infty} t^{A-I} e^{-(I t+(B / t))} \mathrm{d} t, t^{A-I}=\exp ((A-I) \ln t),
$$

for $B=\mathbf{0}$ reduces gamma matrix function in [23].

Also, Abdalla and Bakhet [14] considered the extension of Euler's beta matrix function in the following definition.

Definition 3. Suppose that $A, B$, and $\mathbb{P}$ are positive stable and commutative matrices in $\mathbb{C}^{r \times r}$ satisfying the spectral condition (3); then, the extended Beta matrix function $\mathscr{B}(A, B ; \mathbb{P})$ is defined by

$$
\mathscr{B}(A, B ; \mathbb{P}):=\int_{0}^{1} t^{A-I}(1-t)^{B-I} \exp \left(\frac{-\mathbb{P}}{t(1-t)}\right) \mathrm{d} t .
$$

Hence,

$$
\mathscr{B}(A, B ; \mathbb{P})=\Gamma(A, \mathbb{P}) \Gamma(B, \mathbb{P}) \Gamma^{-1}(A+B ; \mathbb{P}) .
$$

For $\mathbb{P}=\mathbf{0}$, it obviously reduces to the Beta matrix function in $[23,24]$ by

$$
\mathfrak{B}(A, B)=\int_{0}^{1} t^{A-I}(1-t)^{B-I} \mathrm{~d} t .
$$

The Bessel matrix function $J_{A}(z)$ of the first kind associate to $A$ is defined in the form (see $[21,25]$ )

$$
J_{A}(z)=\sum_{k=0}^{\infty} \frac{(-1)^{k}}{(k) !} \Gamma^{-1}(A+(k+1) I)\left(\frac{z}{2}\right)^{A+2 k I}
$$

and the modified Bessel matrix function $I_{A}(z)$ has been defined in the form

$$
I_{A}(z)=\sum_{k=0}^{\infty} \frac{1}{(k) !} \Gamma^{-1}(A+(k+1) I)\left(\frac{z}{2}\right)^{A+2 k I},
$$

where $A$ is a matrix in $\mathbb{C}^{r \times r}$ satisfying condition (3). We can rewrite the Bessel and modified Bessel matrix functions as

$$
\begin{aligned}
& J_{A}(z)=\left(\frac{z}{2}\right)^{A} \Gamma^{-1}(A+I)_{0} F_{1}\left(-; A+I, \frac{-z^{2}}{4}\right), \\
& I_{A}(z)=\left(\frac{z}{2}\right)^{A} \Gamma^{-1}(A+I)_{0} F_{1}\left(-; A+I, \frac{z^{2}}{4}\right),
\end{aligned}
$$

where ${ }_{0} F_{1}\left(-; A+I,\left(-z^{2} / 4\right)\right)$ is a hypergeometric matrix function of 1-denominator [26]:

$$
{ }_{0} F_{1}\left(-; A+I ; \frac{-z^{2}}{4}\right)=\sum_{n \geq 0} \frac{\left[(A+I)_{n}\right]^{-1}}{n !}\left(\frac{-z^{2}}{4}\right)^{n}
$$

and ${ }_{0} F_{1}\left(-; A+I, z^{2} / 4\right)$ is similar.

\section{Generalized Incomplete Gamma Matrix Function}

Definition 4. Let $A$ and $B$ be positive stable matrices in $\mathbb{C}^{r \times r}$ and $x$ be a positive real number. Then, the generalized incomplete Gamma matrix function $\gamma(A, B ; x)$ and its complement $\Gamma(A, B ; x)$ are defined by

$$
\begin{gathered}
\gamma(A, B ; x):=\int_{0}^{x} t^{A-I} e^{-(I t+B / t)} \mathrm{d} t, t^{A-I}=\exp ((A-I) \ln t), \\
\Gamma(A, B ; x):=\int_{x}^{\infty} t^{A-I} e^{-(I t+B / t)} \mathrm{d} t, t^{A-I}=\exp ((A-I) \ln t) .
\end{gathered}
$$

Taking $B=\mathbf{0}$ in (17) and (18), we get the results as [21] 


$$
\begin{aligned}
& \gamma(A, \mathbf{0} ; x)=\gamma(A ; x), \\
& \Gamma(A, \mathbf{0} ; x)=\Gamma(A ; x),
\end{aligned}
$$

where $\gamma(A ; x)$ and $\Gamma(A ; x)$ are defined in (5) and (6).

Theorem 1. Let $A$ and $B$ be positive stable matrices in $\mathbb{C}^{r \times r}$; then, each of the following properties holds true:

(i) $\gamma(A, B ; x)+\Gamma(A, B ; x)=2 \exp (A / 2 \ln B) K_{A}(2 \sqrt{B})$

(ii) $\int_{x}^{\infty} t^{A-I} \exp (-(\alpha t I+B / t)) d t=$

$(\alpha)^{-A^{x}} \Gamma(A, \alpha B ; \alpha x) ; \alpha>0$

\section{Proof}

(i) The following is obtained from Definition 4 and using equation (18)

(ii) The left-hand side equals

$$
\int_{x}^{\infty} t^{A-I} \exp \left(-\left(\alpha t I+\frac{B}{t}\right)\right) \mathrm{d} t
$$

Substituting $t=\tau / \alpha$ and $\mathrm{d} t=\mathrm{d} \tau / \alpha, \alpha>0$, we get that lefthand side becomes

$$
\alpha^{-A} \int_{\alpha x}^{\infty} \tau^{A-I} \exp \left(-\left(\tau I+\alpha \frac{B}{\tau}\right)\right) \mathrm{d} \tau=(\alpha)^{-A} \Gamma(A, \alpha B ; \alpha x),
$$

which is the right-hand side.

For the properties of the generalized incomplete Gamma matrix function, we have these results.

Theorem 2. The generalized incomplete Gamma matrix function $\Gamma(A, B ; x)$ satisfies the following properties:

(i) $\Gamma(A+I, B ; x)=A \Gamma(A, B ; x)+B \Gamma(A-I, B ; x)+$ $x^{A} \exp (-(x I+B / x))$

(ii) $\Gamma(A+I ; x)=A \Gamma(A ; x)+x^{A} \exp (x)$

(iii) $(\partial / \partial B) \Gamma(A, B ; x)=-\Gamma(A-I, B ; x)$

(iv) $\Gamma(A, B ; x)=\sum_{0}^{\infty} \Gamma(A-n I ; x)(-B)^{n} / n !(x>0)$

Proof

(i) Let us define $f(t):=e^{-\left(t I+B t^{-1}\right)} H(t-x)$, where

$$
H(t-x)= \begin{cases}1, & t>x, \\ 0, & t<x,\end{cases}
$$

where $H(t-x)$ is the Heaviside step function; using the Mellin transform of $f(t)$, we obtain

$$
\Gamma(A, B ; x):=\{M f(t) ; A\} .
$$

The differentiation of $f(t)$ is given by

$f^{\prime}(t)=\left(-I+B t^{-2}\right) f(t)+e^{-\left(t I+B t^{-1}\right)} \delta(t-x)$,

where $\delta$ is the Dirac delta function.

From the relation,

$M\left\{f^{\prime}(t) ; A\right\}=-(A-I) M(f(t)(A-I))$,

and between the Mellin transform of a function and derivative, we see that

$-(A-I) \Gamma(A-I, B ; x)=-\Gamma(A, B ; x)+B \Gamma(A-2 I ; B ; x)$

$$
+x^{A-I} \exp -\left(x I+B x^{-1}\right) \text {. }
$$

Replacing $A$ by $A+I$ in (27), we get the proof of (i).

(ii) This follows from (i) when we put $B=\mathbf{0}$.

(iii) From the definition of the generalized incomplete Gamma matrix function, we have

$$
\begin{aligned}
\frac{\partial}{\partial B}(\Gamma(A, B ; x)) & =\frac{\partial}{\partial B}\left(\int_{x}^{\infty} t^{A-I} e^{-(I t+B / t)}\right) \mathrm{d} t \\
& =-\int_{x}^{\infty} t^{A-2 I} e^{-(I t+B / t)} \mathrm{d} t \\
& =-\Gamma(A-I, B ; x) .
\end{aligned}
$$

(iv) Replacing $e^{-B / t}$ in (18) by its series representation yields the series

$$
\Gamma(A, B ; x)=\sum_{n=0}^{\infty} \frac{-B^{n}}{n !} \int_{x}^{\infty} t^{A-(n+1) I} \mathrm{~d} t,
$$

which is exactly (iv).

Theorem 3. For the generalized incomplete Gamma matrix function $\Gamma(A, B ; x)$, we have the following integral:

$$
\begin{aligned}
& \int_{0}^{t} \Gamma\left(A, B ; \frac{1}{x}\right) \exp ((B-C) x) \mathrm{d} x \\
= & (B-C)^{-1} \exp (-t C)\left[\Gamma\left(A, B ; \frac{1}{t}\right) \exp (t B)-\Gamma\left(A, C ; \frac{1}{t}\right) \exp (t C)\right] .
\end{aligned}
$$


Proof. According to (18), we have

$$
\Gamma\left(A, B ; \frac{1}{t}\right)=\int_{1 / t}^{\infty} \tau^{A-I} e^{-(\tau I+B / \tau)} \mathrm{d} \tau .
$$

Substituting $\tau=1 / \zeta$ in (31), we obtain

$$
\Gamma\left(A, B ; \frac{1}{t}\right)=\int_{0}^{t} e^{-B \zeta e^{-I / \zeta} \zeta^{(A+I)} \mathrm{d} \zeta} .
$$

Multiplying both the sides in (32) by $e^{B t}$, we find that

$$
e^{t B} \Gamma\left(A, B ; \frac{1}{t}\right)=\int_{0}^{t} e^{B(t-\zeta)} e^{-I / \zeta} \zeta^{-(A+I)} \mathrm{d} \zeta
$$

which can be written in the convolution operator form as

$$
e^{t B} \Gamma\left(A, B ; \frac{1}{t}\right)=\left\{e^{t B}\right\} *\left\{e^{-I / t} t^{-(A+I)}\right\} .
$$

Taking the convolution operator of both the side in (34) with $\left\{e^{C t}\right\}$ and using the associative property of convolution, it follows that

$$
\left\{e^{t C}\right\} *\left\{e^{t B} \Gamma\left(A, B ; \frac{1}{t}\right)\right\}=\left(\left\{e^{t C}\right\} *\left\{e^{t B}\right\}\right) *\left\{e^{(-I / t)} t^{-(A+I)}\right\} .
$$

However,

$$
\left\{e^{t C}\right\} *\left\{e^{t B}\right\}=(B-C)^{-1}\left[\left\{e^{t B}\right\}-\left\{e^{t C}\right\}\right]
$$

from (35) and (36) and using (34), we obtain

$$
\begin{aligned}
& e^{t C} \int_{0}^{t} \Gamma\left(A, B ; \frac{1}{x}\right) \exp ((B-C) x) \mathrm{d} x=(B-C)^{-1} \\
& \times\left[\Gamma\left(A, B ; \frac{1}{t}\right) \exp (t B)-\Gamma\left(A, C ; \frac{1}{t}\right) \exp (t C)\right] .
\end{aligned}
$$

The multiplication of both sides in (37) by $e^{-C t}$ yields the proof of Theorem 3.

\section{Extended Incomplete Beta Matrix Function}

Definition 5. Let $A$ and $B$ be positive stable and commuting matrices in $\mathbb{C}^{r \times r}$ satisfying the spectral condition (3) and $x$ be a positive real number; then, the incomplete Beta matrix function $\mathfrak{B}_{x}(A, B)$ is defined in the form

$$
\mathfrak{B}_{x}(A, B):=\int_{0}^{x} t^{A-I}(1-t)^{B-I} \mathrm{~d} t, \quad 0<x<1 .
$$

Now, we consider some properties of the incomplete Beta matrix function; we have the following theorem.

Theorem 4. The incomplete Beta matrix function $\mathfrak{B}_{x}(A, B)$ satisfies the following properties:
(i) $\mathfrak{B}_{x}(A, B)=\mathfrak{B}(A, B)-\mathfrak{B}_{1-x}(B, A)$
(ii) $\mathfrak{B}_{x}(A, B)+\mathfrak{B}_{1-x}(B, A)=\mathfrak{B}(A, B)$
(iii) $\mathfrak{B}_{x}(A, B)=\mathfrak{B}_{x}(A+I, B)+\mathfrak{B}_{x}(A, B+I)$

Proof. (i) the right-hand side of (i), we obtain

$$
\int_{1-x}^{1} u^{B-I}(1-u)^{A-I} \mathrm{~d} u
$$

Putting $u=1-t$, we have

$$
\int_{0}^{x} t^{A-I}(1-t)^{B-I} \mathrm{~d} t=\mathfrak{B}_{x}(A, B) .
$$

(ii) (ii) can obviously be obtained from (i).

(iii) the right-hand side of (iii), we obtain

$$
\int_{0}^{x}\left[t^{A}(1-t)^{B-I}+t^{A-I}(1-t)^{B}\right] \mathrm{d} t,
$$

which, after simple algebraic manipulation, yields

$$
\int_{0}^{x} t^{A-I}(1-t)^{B-I} \mathrm{~d} t=\mathfrak{B}_{x}(A, B) .
$$

Definition 6. Let $A, B$, and $\mathbb{P}$ be positive stable and commuting matrices in $\mathbb{C}^{r \times r}$ satisfying the spectral condition (3) and $x$ be a positive real number. Then, the extended incomplete Beta matrix function $\mathscr{B}_{x}(A, B ; \mathbb{P})$ is defined in the form

$$
\mathscr{B}_{x}(A, B ; \mathbb{P}):=\int_{0}^{x} t^{A-I}(1-t)^{B-I} \exp \left(\frac{-\mathbb{P}}{t(1-t)}\right) \mathrm{d} t, \quad 0<x<1 .
$$

Theorem 5. The extended incomplete Beta matrix function $\mathscr{B}_{x}(A, B ; \mathbb{P})$ satisfies the following integral representations:

$$
\begin{aligned}
& \text { (i) } \mathscr{B}_{x}(A, B ; \mathbb{P})=2 \int_{0}^{T}(\sin u)^{2 A-I} \\
& (\cos u)^{2 B-I} \exp \left(-\mathbb{P} \sec ^{2} u \csc ^{2} u\right) d u,(0<T= \\
& \left.\quad \sin ^{-1}(\sqrt{x}) \leq \pi / 2\right) \\
& \text { (ii) } \mathscr{B}_{x}(A, B ; \mathbb{P})= \\
& e^{-2 \mathbb{P}} \int_{0}^{x} u^{A-I}(1+u)^{-(A+B)} \exp (-\mathbb{P}(u+ \\
& \left.\left.u^{-1}\right)\right) d u,(0<T=(x / 1-x)<\infty)
\end{aligned}
$$

Proof. All cases are straightforward. In particular, (i) follows when we use the transformation $t=\sin ^{2} u$ in (43). The transformation $t=u / 1+u$ in (43) yields (ii).

Then, we consider some properties of the extended incomplete Beta matrix function, and we get the following theorem.

Theorem 6. The extended incomplete Beta matrix function $\mathscr{B}_{x}(A, B ; \mathbb{P})$ satisfies the following properties:

(i) $\mathscr{B}_{x}(A+I, B ; \mathbb{P})+\mathscr{B}_{x}(A, B+I ; \mathbb{P})=\mathscr{B}_{x}(A, B ; \mathbb{P})$

(ii) $\mathscr{B}(A, B ; \mathbb{P})-\mathscr{B}_{1-x}(B, A ; \mathbb{P})=\mathscr{B}_{x}(A, B ; \mathbb{P})$

(iii) $\mathscr{B}_{x}(A, B ; \mathbb{P})+\mathscr{B}_{1-x}(B, A ; \mathbb{P})=\mathscr{B}(A, B ; \mathbb{P})$

(iv) $\mathscr{B}_{x}(A, I-B ; \mathbb{P})=\sum_{n=0}^{\infty}\left((B)_{n} / n !\right), \mathscr{B}_{x}(A+n I, I ; \mathbb{P})$

(v) $\mathscr{B}_{x}(A, B ; \mathbb{P})=\sum_{n=0}^{\infty} \mathscr{B}_{x}(A+n I, B+I ; \mathbb{P})$

Proof. (i) From the left-hand side, we obtain 


$$
\int_{0}^{x}\left[t^{A-I}(1-t)^{B}+t^{A}(1-t)^{B-I}\right] \exp \left(\frac{-\mathbb{P}}{t(1-t)}\right) \mathrm{d} t,
$$

which, after simple algebraic manipulation, yields

$$
\int_{0}^{x} t^{A-I}(1-t)^{B-I} \exp \left(\frac{-\mathbb{P}}{t(1-t)}\right) \mathrm{d} t=\mathscr{B}_{x}(A, B ; \mathbb{P}) .
$$

(ii) From the left-hand side, we obtain

$$
\int_{1-x}^{1} u^{B-I}(1-u)^{A-I} \exp \left(\frac{-\mathbb{P}}{t(1-t)}\right) \mathrm{d} u
$$

Putting $u=1-t$, we have

$$
\int_{0}^{x} t^{A-I}(1-t)^{B-I} \exp \left(\frac{-\mathbb{P}}{t(1-t)}\right) \mathrm{d} t=\mathscr{B}_{x}(A, B ; \mathbb{P}) .
$$

(iii) It is obvious that (iii) can be obtained from (ii).

(iv) Notice that

$$
\begin{aligned}
(1-t)^{-B} & =\sum_{n=0}^{\infty} \frac{(B)_{n}}{n !} t^{n}, \quad|t|<1, \\
\mathscr{B}_{x}(A, I-B ; \mathbb{P}) & =\int_{0}^{x} t^{A-I}(1-t)^{I-B-I} \exp \left(\frac{-\mathbb{P}}{t(1-t)}\right) \mathrm{d} t \\
& =\int_{0}^{x} t^{A-I} \sum_{n=0}^{\infty} \frac{(B)_{n}}{n !} t^{n} \exp \left(\frac{-\mathbb{P}}{t(1-t)}\right) \mathrm{d} t \\
& =\sum_{n=0}^{\infty} \frac{(B)_{n}}{n !} \int_{0}^{x} t^{A(n-1) I} \exp \left(\frac{-\mathbb{P}}{t(1-t)}\right) \mathrm{d} t \\
& =\sum_{n=0}^{\infty} \frac{(B)_{n}}{n !} \mathscr{B}_{x}(A+n I, I ; \mathbb{P}) .
\end{aligned}
$$

Thus, it is asserted by (iv).

(v) Replacing $(1-t)^{B-I}$ in (43) by its series representation,

$$
(1-t)^{B-I}=(1-t)^{B} \sum_{n=0}^{\infty} t^{n}
$$

we obtain

$$
\mathscr{B}_{x}(A, B ; \mathbb{P})=\int_{0}^{x}(1-t)^{B} \sum_{n=0}^{\infty} t^{A+(n-1) I} \exp \left(\frac{-\mathbb{P}}{t(1-t)}\right) \mathrm{d} t .
$$

Interchanging the order of the integration and the summation and using (43) yields the desired result (v).

Remark 1. If $B=A$ and $x=1 / 2$ in (iii) of Theorem 5 , we find that

$$
\mathscr{B}_{1 / 2}(A, A ; \mathbb{P})=\frac{1}{2} \mathscr{B}(A, A ; \mathbb{P}),
$$

which can be further written in terms of the Whittaker matrix function (see [14]) to give

$$
\mathscr{B}_{1 / 2}(A, A ; \mathbb{P})=\sqrt{\pi} 2^{-(A+I)} \mathbb{P}^{A-I / 2} e^{-2 \mathbb{P}} W_{(-A / 2),(A / 2)}(4 \mathbb{P}), \quad \mu(\mathbb{P})>0 .
$$

In particular, when $A=1 / 2 I$, we see that

$$
\mathscr{B}_{1 / 2}\left(\frac{1}{2} I, \frac{1}{2} I ; \mathbb{P}\right)=\frac{\pi}{2}(2 \sqrt{\mathbb{P}}), \quad \mu(\mathbb{P})>0 .
$$

\section{Incomplete Bessel Matrix Function}

In this section, we obtain the application of the incomplete Bessel matrix function (IBMF). First, we give some definitions. 
Definition 7. Let $A$ be matrix in $\mathbb{C}^{r \times r}$, satisfying condition (3); then, the incomplete confluent hypergeometric matrix function (ICHMF) of 1-denominator is defined in the form

$$
{ }_{0} F_{1, x}(-; C ; z)=I+z \sum_{k=0}^{\infty}\left[(2 I)_{k}\right]^{-1} \frac{\mathscr{B}_{x}((k+1) I, C) z^{k}}{k !}
$$

By using integral representation of the incomplete beta matrix function given by (7), then we can obtain the integral representation of the ICHMF as

$$
\begin{aligned}
{ }_{0} F_{1, x}(-; C ; z) & =I+z \sum_{k=0}^{\infty} \frac{\left[(2 I)_{k}\right]^{-1} z^{k}}{k !} \int_{0}^{x} t^{k}(1-t)^{C-I} \mathrm{~d} t . \\
& =I+z \int_{0}^{t}(1-t)^{C-I}{ }_{0} F_{1}(-; 2 I ; t z) \mathrm{d} t
\end{aligned}
$$

Now, we give definitions of an incomplete Bessel matrix function (IBMF) by using ICHMF.

Definition 8. Let $A$ be matrix in $\mathbb{C}^{r \times r}$, satisfying condition (3); then, the incomplete Bessel matrix function IBMF is defined in the form

$$
J_{A, x}(z)=\left(\frac{z}{2}\right)^{A} \Gamma^{-1}(A+I)_{0} F_{1, x}\left(-; A+I, \frac{-z^{2}}{4}\right)
$$

where ${ }_{0} F_{1, x}\left(-; A+I,\left(-z^{2} / 4\right)\right)$ is incomplete hypergeometric matrix function of 1-denominator. By using equation (55), we can easily obtain series representations for the IBMF as

$$
J_{A, x}(z)=\left(\frac{z}{2}\right)^{A} \Gamma^{-1}(A+I)\left(I-\frac{z^{2}}{4} \sum_{k=0}^{\infty} \frac{(-1)^{k} \mathscr{B}_{x}((k+1) I, A+I)(z / 2)^{2 k}}{(2)_{k} k !}\right)
$$

and the integral representations are found from (54) and (55) to obtain

$$
J_{A, x}(z)=\left(\frac{z}{2}\right)^{A} \Gamma^{-1}(A+I)\left(I-\frac{z}{2} \int_{0}^{x} t^{(-1 / 2)}(1-t)^{A} J_{I}(z \sqrt{t}) \mathrm{d} t\right)
$$

Remark 2. (i) If $u=z \sqrt{t}$ is used in (58), we have

$$
J_{A, x}(z)=\left(\frac{z}{2}\right)^{A} \Gamma^{-1}(A+I)\left(I-\int_{0}^{z \sqrt{x}}\left(1-\frac{u^{2}}{z^{2}}\right)^{A} J_{I}(u) \mathrm{d} u\right)
$$

(ii) Putting $A=\mathbf{0}$ in (59), we have

$$
J_{0, x}(z)=I-\int_{0}^{z \sqrt{x}} J_{I}(u) \mathrm{d} u
$$

(iii) By differentiating both sides of (60) with respect to $z$, we get the following elegant relation between the IBMF and the BMF as follows:

$$
J_{0, x}^{\prime}(z)=J_{I}(z \sqrt{x})
$$

The following two theorems give the integrals of the ICHMF and the IBMF over the unit interval as a direct elegant relation with the complete version of both of them, respectively.

Theorem 7. Let $A$ be matrix in $\mathbb{C}^{r \times r}$; we have the relation between the ICHMF and CHMF as follows:

$$
\int_{0}^{1}{ }_{0} F_{1, x}(-; A ; z) \mathrm{d} t={ }_{0} F_{1}(-; A+I ; Z) .
$$

Proof. By using (54) and integrating both sides over the unit interval, we obtain 


$$
\int_{0}^{1}{ }_{0} F_{1, x}(-; A ; z) \mathrm{d} t=I+z \int_{0}^{1} \sum_{k=0}^{\infty}\left[(2 I)_{k}\right]^{-1} \frac{\mathscr{B}_{x}((k+1) I, A) z^{k}}{k !} \mathrm{d} t
$$

since the integral of the unity over the unit interval is again one. So, as before, interchanging the integral sign with the summation sign gives

$$
\int_{0}^{1}{ }_{0} F_{1, x}(-; A ; z) \mathrm{d} t=I+z \int_{0}^{1} \sum_{k=0}^{\infty} \frac{z^{k}\left[(2 I)_{k}\right]^{-1}}{k !} \int_{0}^{1} \mathscr{B}_{x}((k+1) I, A) \mathrm{d} t .
$$

Now, by using properties of the integral in compleat Beta matrix function, we find that

$$
\begin{aligned}
\mathscr{B}_{x}((k+1) I, A) & =\mathscr{B}((k+1) I, A+I), \\
\int_{0}^{1}{ }_{0} F_{1, x}(-; A ; z) \mathrm{d} t & =I+z \sum_{k=0}^{\infty}\left[(2 I)_{k}\right]^{-1} \frac{\mathscr{B}_{x}((k+1) I, A+I) z^{k}}{k !},
\end{aligned}
$$

which is the required proof.

Theorem 8. Let $A$ be matrix in $\mathbb{C}^{r \times r}$, and we have the relation between the IBMF and BMF as follows:

$$
\int_{0}^{1} J_{A, x}(z) \mathrm{d} t=\frac{2(A+I)}{z} J_{A+I}(z) .
$$

Proof. From definition of an incomplete Bessel matrix function, we have

$$
\int_{0}^{1} J_{A, x}(z) \mathrm{d} t\left(\frac{z}{2}\right)^{A} \Gamma^{-1}(A+I) \int_{0}^{1}{ }_{0} F_{1, x}\left(-; A+I, \frac{-z^{2}}{4}\right) .
$$

By (62) and (68), it is written as

$$
\int_{0}^{1} J_{A, x}(z) \mathrm{d} t=\left(\frac{z}{2}\right)^{A} \Gamma^{-1}(A+I) \int_{0}^{1}{ }_{0} F_{1}\left(-; A+2 I, \frac{-z^{2}}{4}\right) .
$$

By properties of Bessel matrix function, we obtain

$$
{ }_{0} F_{1}\left(-; A+2 I, \frac{-z^{2}}{4}\right)=\left(\frac{z}{2}\right)^{-(A+I)} \Gamma(A+2 I) J_{A+I}(z) .
$$

Hence, substitution of (70) in (69) completes the proof of Theorem 8 desired.

\section{Conclusions}

Special matrix functions play an important role in certain parts of mathematics and physics over the last years. In this paper, we have introduced the incomplete extended Gamma and Beta functions with matrix parameters; then, we establish some properties for these new extension; furthermore, we give a special application for the incomplete Bessel matrix function by using incomplete extended Gamma and Beta functions; at last, we construct the relation between the incomplete confluent hypergeometric matrix functions and incomplete Bessel matrix function. Taking $B=0$ in (17) and (18), we get the results in [21].

Also, by using the identity matrix, we can find the scalar case incomplete extended Gamma and Beta functions in $[1,27-30]$ as $A=\alpha I$ and $B=\beta I$. If $A=\alpha I$ and $B=\mathbf{0}$, we have Gamma and Beta functions.

\section{Conflicts of Interest}

The authors declare that they have no conflicts of interest.

\section{Acknowledgments}

This research was supported by Educational Science "13th Five Year Plan" Project Foundation of Hunan Province (XJK19BXX005) and Natural Science Foundation of Hunan Province (2020JJ4105).

\section{References}

[1] P. Agarwal, S. Dragomir, M. Jleli, and B. Samet, Advances in Mathematical Inequalities and Applications (Trends in Mathematics), Birkhäser, Basel, Switzerland, 2019.

[2] P. Agarwal, J. Choi, and S. Jain, "Extended hypergeometric functions of two and three variables," Communications of the Korean Mathematical Society, vol. 30, no. 4, pp. 403-414, 2015. 
[3] P. Agarwal, M. Chand, and S. D. Purohit, "A note on generating functions involving the generalized Gauss hypergeometric functions," National Academy Science Letters, vol. 37, no. 5, pp. 457-459, 2014.

[4] F. He, A. Bakhet, M. Akel, and M. Abdalla, "Degenerate analogues of euler zeta, digamma, and polygamma functions," Mathematical Problems in Engineering, vol. 2020, Article ID 8614841, 9 pages, 2020.

[5] M. Ruzhansky, Y. JeCho, P. Agarwal, and I. Area, Advances in Real and Complex Analysis with Applications (Trends in Mathematics), Birkhaser, Basel, Switzerland, 2017.

[6] R. Srivastava, "Some classes of generating functions associated with a certain family of extended and generalized hypergeometric functions," Applied Mathematics and Computation, vol. 243, pp. 132-137, 2014.

[7] H. M. Srivastava, P. Agarwal, and S. Jain, "Generating functions for the generalized Gauss hypergeometric functions," Applied Mathematics and Computation, vol. 247, pp. 348-352, 2014.

[8] M. Abul-Dahab, M. Abul-Ez, Z. Kishka, and D. Constales, "Reverse generalized Bessel matrix differential equation, polynomial solutions, and their properties," Mathematical Methods in the Applied Sciences, vol. 38, no. 6, pp. 1005-1013, 2015.

[9] A. M. Mathai and H. Haubold, Special Functions for Applied Scientists, Springer Science, New York, NY, USA, 2008.

[10] A. M. Mathai, A Handbook of Generalized Special Functions for Statistical and Physical Sciences, Oxford University Press, Oxford, UK, 1993.

[11] W. Miller, Lie Theory and Specials Functions, Academic Press, New York, NY, USA, 1968.

[12] M. Abdalla, "Further results on the generalised hypergeometric matrix functions," International Journal of Computing Science and Mathematics, vol. 10, no. 1, pp. 1-10, 2019.

[13] M. Abul-Dahab and A. Bakhet, "A certain generalized gamma matrix functions and their properties," Journal of Analysis and Number Theory, vol. 3, pp. 63-68, 2015.

[14] M. Abdalla and A. Bakhet, "Extension of beta matrix function," Journal of Mathematics and Computer Research, vol. 9, pp. 253-264, 2016.

[15] M. Abdalla and A. Bakhet, "Extended Gauss hypergeometric matrix functions," Iranian Journal of Science and Technology, Transactions A: Science, vol. 42, no. 3, pp. 1465-1470, 2018.

[16] M. Abdalla, "On the incomplete hypergeometric matrix functions," The Ramanujan Journal, vol. 43, no. 3, pp. 663-678, 2017.

[17] A. Bakhet, Y. Jiao, and F. He, "On the wright hypergeometric matrix functions and their fractional calculus," Integral Transforms and Special Functions, vol. 30, no. 2, pp. 138-156, 2019.

[18] A. Bakhet and F. He, "On 2-variables Konhauser matrix polynomials and their fractional integrals," Mathematics, vol. 8 , no. 2, p. 232, 2020.

[19] F. He, A. Bakhet, M. Abdalla, and M. Hidan, "On the extended hypergeometric matrix functions and their applications for the derivatives of the extended Jacobi matrix polynomial," Mathematical Problems in Engineering, vol. 2020, Article ID 4268361, 8 pages, 2020.

[20] A. Bakhet, F. He, and Mimi Yu, "On the matrix version of extended Bessel functions and its application to matrix differential equations," Linear and Multilinear Algebra, 2021.

[21] J. Sastre and L. Jódar, "Asymptotics of the modified bessel and the incomplete gamma matrix functions," Applied Mathematics Letters, vol. 16, no. 6, pp. 815-820, 2003.
[22] M. Abdalla, "Special matrix functions: characteristics, achievements and future directions," Linear and Multilinear Algebra, vol. 68, no. 1, pp. 1-28, 2020.

[23] L. Jódar and J. C. Cortés, "Some properties of Gamma and Beta matrix functions," Applied Mathematics Letters, vol. 11, no. 1, pp. 89-93, 1998 .

[24] F. He, A. Bakhet, M. Hidan, and M. Abdalla, "Two variables Shivley's matrix polynomials," Symmetry, vol. 11, no. 2, p. 151, 2019.

[25] L. Jódar, R. Company, and E. Navarro, "Solving explicitly the Bessel matrix differential equation, without increasing problem dimension," Congressus Numerantium, vol. 92, pp. 261-276, 1993.

[26] L. Jódar and J. C. Cortés, "On the hypergeometric matrix function," Journal of Computational and Applied Mathematics, vol. 99, no. 1-2, pp. 205-217, 1998.

[27] F. Ayant and D. Kumar, "Generating relations and multivariable Aleph-function," Analysis, vol. 38, no. 3, pp. 137-143, 2018.

[28] R. S. Batahan, "Study and developing some subjects in complex analysis," Ph. D. Thesis, Assiut University, Asyut, Egypt, 2004.

[29] J. Choi and P. Agarwal, "Certain integral transform and fractional integral formulas for the generalized Gauss hypergeometric functions," Abstract and Applied Analysis, vol. 2014, Article ID 735946, 2014.

[30] M.-J. Luo, G. V. Milovanovic, and P. Agarwal, "Some results on the extended beta and extended hypergeometric functions," Applied Mathematics and Computation, vol. 248, pp. 631-651, 2014 